OPEN ACCESS

Edited by:

Nuno Madeira,

University of Coimbra, Portugal

Reviewed by:

Bruno Manadas,

University of Coimbra, Portugal

Cátia Santa,

University of Coimbra, Portugal

*Correspondence:

Yan Ren

renyansxmu@outlook.com

${ }^{\dagger}$ These authors share first authorship

Specialty section: This article was submitted to Mood Disorders,

a section of the journal

Frontiers in Psychiatry

Received: 27 July 2021 Accepted: 24 November 2021 Published: 17 December 2021

Citation

Guo X-J, Wu P, Cui X-H, Jia J, Bao S,

Yu F, Ma L-N, Cao X-X and Ren Y (2021) Pre- and Post-treatment Levels of Plasma Metabolites in Patients With Bipolar Depression Front. Psychiatry 12:747595 doi: 10.3389/fpsyt.2021.747595

\section{Pre- and Post-treatment Levels of Plasma Metabolites in Patients With Bipolar Depression}

\author{
Xiang-Jie Guo ${ }^{1 \dagger}$, Peng $\mathrm{Wu}^{1 \dagger}$, Xiao-Hong Cui ${ }^{2,3}$, Jiao Jia ${ }^{2,3}$, Shuang Bao ${ }^{2,3}, \mathrm{Fei} \mathrm{Yu}^{2,3}$, \\ Li-Na Ma ${ }^{2,3}$, Xiang-Xin $\mathrm{Cao}^{2,3}$ and Yan $\mathrm{Ren}^{2,3 *}$ \\ ${ }^{1}$ Department of Forensic Medicine, Shanxi Medical University, Taiyuan, China, ${ }^{2}$ Department of Psychiatry, Shanxi Bethune \\ Hospital, Shanxi Academy of Medical Sciences, Tongji Shanxi Hospital, Third Hospital of Shanxi Medical University, Taiyuan, \\ China, ${ }^{3}$ Tongji Hospital, Tongji Medical College, Huazhong University of Science and Technology, Wuhan, China
}

Background: Bipolar disorder $(\mathrm{BD})$ is a serious mental disease with complex clinical manifestations and high recurrence rate. The purpose of this study was to detect metabolites related to the diagnosis and efficacy evaluation of bipolar depression in plasma samples by metabolomics.

Methods: Thirty-one bipolar depression patients were recruited and completed 8 weeks medication and a matched group of 47 healthy controls (HCs) was recruited. Nuclear magnetic resonance spectroscopy was used to profile plasma samples of bipolar depression patients at baseline and after 8 weeks medication, and HCs. Then Multivariate statistical analysis was performed to analyze differences of plasma metabolites among the three groups.

Results: We detected seven specific differential metabolites in bipolar depression. Six of the metabolites were returned to the normal levels in different degrees after 8 weeks medication, only Glycine continuously decreased in the acute and significant improvement stages of bipolar depression (VIP $>1$ and $p<0.05$ ). These differential metabolites involved several metabolic pathways.

Limitations: The small sample size was one of the most prominent limitations. Each $\mathrm{BD}$ patient was given an individualized medication regimen according to the clinical guidelines.

Conclusion: There were metabolites changes before and after 8 weeks medication. Glycine may be a characteristic marker of bipolar depression and does not change with the improvement of bipolar depression, while other 6 differential metabolites may be biomarkers associated with the pathological development or the improvement of bipolar depression. And, these differential metabolites mainly related to energy metabolism, amino acid metabolism and gut microbiota metabolism.

Keywords: bipolar disorder (BD), bipolar depression, pre- and post-treatment, metabolomics, efficacy evaluation, biomarker 


\section{INTRODUCTION}

$\mathrm{BD}$ is a chronic severe mental illness, characterized by the intermittent occurrence of depression and mania (or hypomania). The average age of onset of BD was 20-30 years (1), with a lifetime prevalence of about $2.4 \%$ (2) and a recurrence rate of $40-50 \%$ within 2 years (3). Patients with BD often suffer from comorbid anxiety, substance abuse and other mental diseases, resulting in great damage to their ability to live, study and work. The vast majority of BD patients need lifelong monitoring and treatment. Due to the multi-etiological complexity of the disease, there are no specific biomarkers to guide clinical diagnosis and the evaluation of drug treatment effectiveness. Researchers have found that BD may be associated with changes in neurotransmitters, immunology, neuroimaging, neuroendocrinology, neuroplasticity, molecular genetics, and adverse environmental stress (4-8), but they have not been conclusively consistent. Therefore, the study of objective markers of $\mathrm{BD}$ has always been a hot and difficult research topic.

Although the pathologic mechanisms of $\mathrm{BD}$ are poorly understood, the research on drugs to treat $\mathrm{BD}$ has made relatively considerable progress. At present, the commonly used emotional stabilizers for the treatment of $\mathrm{BD}$ are lithium, valproate, lamotrigine and some antipsychotics (such as quetiapine and olanzapine) which have the effect of stabilizing emotion. Patients with $\mathrm{BD}$ at the onset of depression may be treated with a combination of antidepressants when mood stabilizers or antipsychotics do not work well. Interestingly, the therapeutic effect of these drugs on BD is mostly the conclusion of clinical observation and research, but their mechanism of action has not been clarified. Myo-inositol depletion is a well-characterized effect of lithium treatment and has also been found to occur after valproate treatment $(9,10)$. Antipsychotics and antidepressants work by acting on specific receptors. These limited findings cannot elucidate the internal mechanism of $\mathrm{BD}$ remission. Therefore, it will be a significant research direction to explore the pathology of $\mathrm{BD}$ from the perspective of the common pathway of the efficacy of drug therapy.

Metabolomics is a research method for qualitative and quantitative analysis of small molecular metabolites in biological samples (11), which is widely used to study the pathological mechanism of complex diseases, such as cancer, diabetes, cardiovascular diseases, and psychiatric diseases, as well as to find new biomarkers for diagnosis and prognosis. Many studies on the metabolomics of $\mathrm{BD}$ have been reported, mainly including the study on the diagnostic biomarkers of $\mathrm{BD}$ and the changes of metabolites related to drug treatment (12-15). These studies have found some significant specific metabolites, which need to be verified by more similar studies. Studies on biomarkers related to the efficacy of drug therapy for $\mathrm{BD}$ are rarely reported, and

\footnotetext{
Abbreviations: BD, Bipolar disorder; HC, healthy control; NMR, Nuclear magnetic resonance; PLS-DA, Partial Least Square Discriminant Analysis; OPLSDA, Orthogonal Partial Least Square Discriminant Analysis; DSM-V, The Diagnostic and Statistical Manual of Mental Disorders V; VIP, Variable importance in the projection; HAMD-24, 24-item Hamilton Depression Rating Scal; HAMA14, 14-item Hamilton Anxiety Rating Scale; CGI, Clinical General Impression Rating Scale; BMI, Body mass index.
}

most of them are in vitro studies, such as animal models or post-mortem brain.

Considering that the depression episode is more pervasive than manic states in the course of BD (16), and the pathological mechanism of these two mood polarities are different, this research focused on bipolar depression. As defined in DSM5 , depression is characterized by at least 2 weeks of depressive mood and/or anhedonia, along with at least four of other possible symptoms that include changes in energy, psychomotor activity, concentration, sleep, appetite/weight, thought content (guilt and worthlessness) and suicidal intent. Our research group has carried out a previous study on diagnostic biomarkers in the blood of bipolar depression and found some differential metabolites (17). We would like to further investigate how the specific differential metabolites of bipolar depression at baseline will change as their condition improves with medication.

Therefore, in this study, patients with bipolar depression, belonging to $\mathrm{BD}$ II type, were treated with medication for 8 weeks, and the changes of plasma metabolites before and after medication were analyzed using ${ }^{1} \mathrm{H}-\mathrm{NMR}$ metabolomics analysis method, so as to screen the metabolites that can be used for the evaluation of drug treatment effectiveness and analyze the internal mechanism of disease improvement by drug therapy.

\section{MATERIALS AND METHODS \\ Subjects Recruitment and Sample Collection}

Ethics approval for this study is held by the medical ethics committee of Shanxi Bethune hospital (the Approval Notice Number: YXLL-2020-001). All subjects enrolled in the study gave their written informed consent. In order to reduce the interference of different types of $\mathrm{BD}$ on the results, the inclusion requirements of the patient group were first to conform to the $\mathrm{BD}$ II type, which is characterized by the intermittent occurrence of threshold depressive episodes and hypomanic episodes that are qualitatively like manic periods but are not of a sufficient duration or severity to cause significant functional impairment, hospitalization, or psychosis. Thirty-one unmedicated patients with bipolar depression were eventually recruited from the outpatient and inpatient of Shanxi Bethune Hospital and completed 8 weeks of drug therapy and follow-up, and 47 healthy subjects matching their demographic characteristics were also recruited from the hospital's physical examination center. All subjects were between 18 and 60 years old. The patients in the bipolar depression group were diagnosed by the Diagnostic and Statistical Manual of Mental Disorders V (DSM-V). Patients with current or previous presence of any physical or other mental disorders, as well as substance abuse were excluded. The subjects in HC group should also have no physical or mental disorders, and substance abuse.

General demographic data, including sex, age, marital status, and body mass index (BMI), were recorded for the patient group and the healthy control group. Subjects with BD were assessed using the 24-item Hamilton Depression Rating Scale (HAMD24), 14-item Hamilton Anxiety Rating Scale (HAMA-14) and 
Clinical General Impression Rating Scale (CGI) at baseline and post- 8 weeks medication. In this study, the formulation of the medication regimens were referred to the pharmacological treatment principles of acute bipolar depression in The Chinese Guidelines for the Prevention and Treatment of Bipolar Disorder (Second Edition) and Canadian Network for Mood and Anxiety Treatments (CANMAT) and International Society for Bipolar Disorders (ISBD) 2018 guidelines for the management of patients with bipolar disorder (18). Single drug therapy was the first choice for the treatment of acute bipolar depression (such as quetiapine, lithium, and Lamotrigine). Once the effect of monotherapy is not ideal, the combination of drugs should be considered, which means the combination of mood stabilizers (such as lithium, Lamotrigine and valproate) and/or atypical antipsychotics (such as quetiapine and olanzapine). Treatment with the combinations of an antidepressant [selective serotonin reuptake inhibitors (SSRIs) or bupropion] on the basis of lithium/divalproex or an atypical antipsychotic is considered second-line medication regimes. Antidepressants should be used with caution in patients with a history of antidepressant-induced mania or hypomania. Efficacy was assessed according to the reduction rate of HAMD (cure: a reduction of HAMD scores by $\mathrm{Z75} \%$; significant progress: a reduction of $Z 50 \%$; progress: a reduction of $Z 25 \%$; ineffective: a reduction of $<\mathrm{Z} 25 \%$ ).

\section{Plasma Collection}

Blood samples of the recruited subjects were collected using vacuum blood collection vessels containing heparin sodium by professionals in the morning (between 07:30 and 10:00) after a 12-h fasting. The blood samples were mixed upside down to prevent coagulation, kept for $0.5 \mathrm{~h}$ at room temperature, and then centrifuged for $15 \mathrm{~min}$ at $1,600 \times \mathrm{g}$. The obtained supernatant was sucked out into a sterile EP tube with a pipette and stored at $-80^{\circ} \mathrm{C}$ until NMR analysis. The plasma of healthy controls was collected once, and the plasma samples of bipolar depression subjects were collected before and after 8 weeks of medication.

\section{Sample Preparation and NMR Procedure}

The plasma samples should be pretreated before NMR analysis. The pre-processing flow is as follows: (1) The plasma samples were removed from the $-80^{\circ} \mathrm{C}$ refrigerator and thawed in the ice water compound; (2) 450 ul plasma samples were extracted with a pipette and mixed with $350 \mathrm{ul}$ phosphate buffer $(0.2 \mathrm{M}$ $\mathrm{Na} 2 \mathrm{HPO} 4 / \mathrm{NaH} 2 \mathrm{PO} 4, \mathrm{pH}=7.4)$ in $\mathrm{D} 2 \mathrm{O}$ containing sodium 3trimethylsilyl-(2, 2, 3, 3-d4)-1-propionate (TSP, 0.01\%); (3) The mixture was centrifuged for $20 \mathrm{~min}$ at $15,493 \times \mathrm{g}$ at $4^{\circ} \mathrm{C}$; (4) The supernatant $(600 \mu \mathrm{l})$ was transferred into $5 \mathrm{~mm}$ NMR tubes for NMR analysis.

The Bruker $600 \mathrm{MHz}$ AVANCE III NMR spectrometer (Bruker Biospin, Rheinstetten, Germany) was used for data acquisition. The ${ }^{1} \mathrm{H}$ NMR spectra of the plasma samples were recorded in a one-dimensional (1D) Carr-Purcell-MerboomGill [CPMG, RD-90-( $\tau \mathrm{cp}-180-\tau \mathrm{cp})$-acquisition] with water suppression. The key parameters for the NMR procedure were as follows: ${ }^{1} \mathrm{H}$ frequency, $600.13 \mathrm{MHz}$; test temperature, $293 \mathrm{~K}$; scanning time/scans, $5 \mathrm{~min} / 64$; spectral width, $12,019.2 \mathrm{~Hz}$; spectral size, 65,536 points; pulse width (PW), 30 (12.7 $\mu \mathrm{s}$ ); relaxation delay (RD), $1.0 \mathrm{~s}$; The free induction decay (FID) was Fourier transformed with a line-broadening factor of $0.3 \mathrm{~Hz}$. The NMR spectra of plasma samples were acquired according to previous relevant literature and NMR databases (19).

\section{Data Processing}

Phase and baseline adjustment of the acquired ${ }^{1} \mathrm{H}$ NMR spectra was performed manually using the MestReNova software (Mestrelab Research, Santiago de Compostella, Spain). The chemical shift of creatinine $(\delta 3.04 \mathrm{ppm})$ was used as a calibration standard. The region of $\delta 4.7-5.2 \mathrm{ppm}$ was excluded due to residual water. Each spectrum was then segmented at $\delta 0.01$ intervals across the region of $\delta 0.5-5.5 \mathrm{ppm}$. These data of integral value were finally normalized by dividing an individual peak area by the total peak area in ${ }^{1} \mathrm{H}$ NMR profile of each subject for further multivariate analysis.

\section{Statistical Analyses}

The processed spectra data were imported into SIMCA-P 14.1 software (Umetric, Sweden) for Orthogonal Partial Least Square Discriminant Analysis (OPLS-DA). The score plot of the OPLSDA model could be used to visualize the differences between group clusters. It is necessary to perform 200 permutation tests and evaluate the validity of the established OPLS-DA model according to the values of $R^{2}$ and $Q^{2}$. The corresponding loading plot of the OPLS-DA model was further established to discover the differential metabolites contributing to samples separation. The differential metabolites were finally identified according to the standard of variable importance in the projection (VIP > 1) in the loading plot and $p<0.05$ of independent samples $t$-test between $\mathrm{BD}$ at baseline and healthy controls (HCs) or $p<0.05$ of paired samples $t$-test between BD patients at baseline and $\mathrm{BD}$ patients after 8 weeks medication using SPSS 25 software.

To determine the pathways involved in relative metabolites, they were further introduced into MetaboAnalyst 4.0 to perform Pathway analysis by selecting the human Pathway library. Pathways were screened according to the $p$-values of pathway enrichment and impact values of pathway topology analysis.

\section{RESULTS}

\section{General Information of Subjects}

Thirty-one patients with bipolar depression were enrolled and completed 8 weeks of medication. Table 1 shows the clinical features of the subjects. Age, gender, BMI and marital status between $\mathrm{BD}$ patients at baseline and $\mathrm{HCs}$ were compared using Chi square test or independent samples $t$-test, and the results indicated no significant differences $(p>0.05)$. The clinical features between BD patients at baseline and BD patients after 8 weeks medication was compared using paired samples $t$ test, and results are shown in Table 1. Depression, anxiety and clinical status of patients with bipolar depression at baseline were shown by HAMD scores, HAMA scores and CGI scores. These patients did not meet the diagnosis of anxiety disorder. After 8 weeks medication, HAMD scores, HAMA scores and CGI scores of patients in $\mathrm{BD}$ group were significantly reduced, and the reduction rate of the three scales was above $50 \%$ which indicated 
TABLE 1 | General characteristics of the subjects, including BD patients at baseline and after 8 weeks medication, and healthy controls.

\begin{tabular}{|c|c|c|c|c|c|}
\hline \multirow[t]{2}{*}{ Clinical features } & \multicolumn{2}{|c|}{$\mathrm{BD}(n=31)^{\mathrm{b}}$} & \multirow[t]{2}{*}{ Healthy controls $(n=47)^{\mathrm{b}}$} & \multirow[b]{2}{*}{$p$-value ${ }^{c}$} & \multirow[b]{2}{*}{$p$-value ${ }^{\star c}$} \\
\hline & Baseline & After 8 weeks medication & & & \\
\hline Age (years) & $30.26 \pm 12.695$ & $--^{d}$ & $30.66 \pm 8.231$ & - & 0.867 \\
\hline Gender(male/female) & $13 / 18$ & - & $23 / 24$ & - & 0.644 \\
\hline BMI $\left(\mathrm{kg} / \mathrm{m}^{2}\right)^{\mathrm{a}}$ & $23.42 \pm 3.766$ & - & $22.31 \pm 3.041$ & - & 0.177 \\
\hline Spouse (with/without) & $19 / 12$ & - & $23 / 24$ & - & 0.355 \\
\hline HAMD-24 total score ${ }^{a}$ & $21.42 \pm 7.86$ & $9.13 \pm 7.293$ & - & 0.000 & - \\
\hline \multicolumn{6}{|l|}{ HAMD factor score } \\
\hline Anxiety/somatization & $5.45 \pm 2.474$ & $2.68 \pm 1.759$ & - & 0.000 & - \\
\hline Weigh reduction & $0.16 \pm 0.374$ & $0.06 \pm 0.250$ & - & 0.235 & - \\
\hline Cognitive impairment & $3.94 \pm 2.351$ & $2.03 \pm 2.008$ & - & 0.001 & - \\
\hline Diurnal variation & $0.71 \pm 1.039$ & $0.29 \pm 0.643$ & - & 0.419 & - \\
\hline Slowness & $4.61 \pm 2.108$ & $2.29 \pm 1.736$ & - & 0.000 & - \\
\hline Sleep disorder & $2.23 \pm 1.687$ & $1.03 \pm 0.875$ & - & 0.001 & - \\
\hline Despair sense & $4.16 \pm 1.791$ & $1.32 \pm 1.720$ & - & 0.000 & - \\
\hline HAMA-14 total score ${ }^{a}$ & $16.58 \pm 6.076$ & $8.00 \pm 5.373$ & - & 0.000 & - \\
\hline \multicolumn{6}{|l|}{ HAMA factor score } \\
\hline Anxiety of somatization & $10.45 \pm 4.146$ & $5.55 \pm 3.641$ & - & 0.000 & - \\
\hline Mental anxiety & $5.48 \pm 2.839$ & $2.65 \pm 2.229$ & - & 0.000 & - \\
\hline CGI score ${ }^{a}$ & $4.55 \pm 0.888$ & $2.90 \pm 0.790$ & - & 0.000 & - \\
\hline \multicolumn{6}{|l|}{ Medicine } \\
\hline Mood stabilizers & $28(90.32 \%)$ & - & - & - & - \\
\hline Lithium & $17(54.84 \%)$ & - & - & - & - \\
\hline Lamotrigine & 9 (29.03\%) & - & - & - & - \\
\hline Sodium valproate & $2(6.45 \%)$ & - & - & - & - \\
\hline Antipsychotics & $22(70.97 \%)$ & - & - & - & - \\
\hline Antidepressants & 7 (22.58\%) & - & - & - & - \\
\hline
\end{tabular}

a HAMD-24, 24-item Hamilton Depression Rating Scale; HAMA-14, 14-item Hamilton Anxiety Rating Scale; CGI, Clinical global impression scale; BMI, Body mass index.

${ }^{b}$ Values expressed as the mean $\pm S D$ (range).

${ }^{c} p$-value represent $B D$ patients at baseline vs. BD patients after 8 weeks medication, and p-value* represent BD at baseline vs. Healthy controls.

d "-" Means that the data are not shown.

significant curative effect and significant improvement of mood symptoms of BD subjects. All factor scores of HAMD and HAMA were also significantly reduced after 8 weeks of drug therapy.

\section{Identification of Metabolites in the ${ }^{\mathbf{1}} \mathrm{H}$-NMR Profiles}

The ${ }^{1} \mathrm{H}$ NMR spectra of the three groups of plasma samples were detected one by one. Figure 1 shows the typical ${ }^{1} \mathrm{H}$ NMR spectrum profiles of $\mathrm{BD}$ patients at baseline and after 8 weeks medication, and HCs. In the typical ${ }^{1} \mathrm{H}$ NMR spectra of the three groups, a total of 22 metabolites were identified by looking up the Human Metabolome Database (HMDB) (http://www.hmdb. $\mathrm{ca} /)$ and related articles over the years $(20,21)$. The chemical shift values of these 22 metabolites in the typical ${ }^{1} \mathrm{H}$ NMR spectra are listed in Table 2.

\section{Multivariate Statistical Analysis}

The integral data of all the plasma spectra were taken into the SIMCA-P 14.1 software (Umetric, Sweden) for multivariate statistical analysis. We established the PLS-DA model to observe vividly metabolites differences of the three groups. The score plot of the PLS-DA model showed clear differentiations among the group of bipolar depression at baseline, the group of bipolar depression after 8 weeks medication and HC group, and that the bipolar depression after 8 weeks medication group was closer to the HC group than bipolar depression at baseline group, suggesting a disturbance of the plasma metabolites in bipolar depression patients and a trend to reversion to the healthy control state after 8 weeks medication (Figure 2A). Permutation testing showed that the cumulative $R^{2}$ - and $Q^{2}$-values were all less than the original value, indicating that the PLS-DA model was not over-fitted (Figure 2B).

To further identify the metabolites changes associated with bipolar depression at baseline and after 8 weeks medication, OPLS-DA was applied in this study. The group of bipolar depression at baseline was significantly separated from HC group in the OPLS-DA scores plot (Figure 3A), which were verified to be valid by the permutation testing (Figure 3B). Consistent with our previous findings, bipolar depression patients at baseline were characterized by significantly higher levels of lactate, and significantly lower levels of Choline, $\alpha$-glucose, $\beta$-glucose, glycine, $\mathrm{N}$-acetyl glycoprotein, trimethylamine oxide compared 


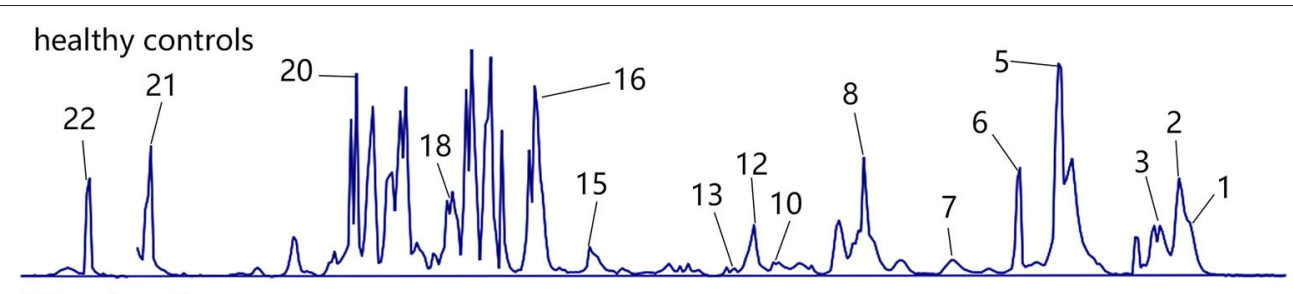

$\mathrm{BD}$ at baseline

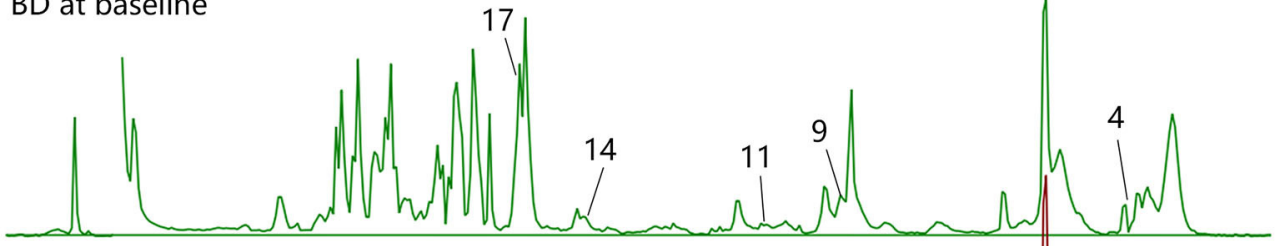

$\mathrm{BD}$ after 8 weeks medication

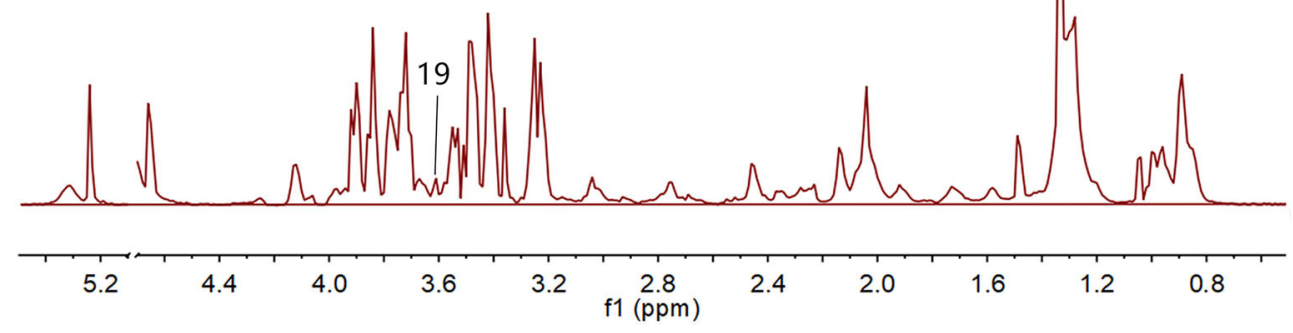

FIGURE 1 | The typical ${ }^{1} \mathrm{H}$ NMR spectra of the three groups of plasma samples.

TABLE 2 | Peak attribution in the typical ${ }^{1} \mathrm{H}-\mathrm{NMR}$ spectra of plasma samples.

\begin{tabular}{|c|c|c|}
\hline No. & Metabolites & Chemical shift $(\delta)$ \\
\hline 1 & Lipid & $0.86(\mathrm{t}), 1.28(\mathrm{~m}), 1.58(\mathrm{~m}), 2.01(\mathrm{~m})$ \\
\hline 2 & Isoleucine & $0.93(\mathrm{t}, J=7.41 \mathrm{~Hz}), 1.01(\mathrm{~d}, J=7.01 \mathrm{~Hz})$ \\
\hline 3 & Leucine & $0.96(\mathrm{t}, \mathrm{J}=7.08 \mathrm{~Hz})$ \\
\hline 4 & Valine & $0.99(\mathrm{~d}, J=8.41 \mathrm{~Hz}), 1.04(\mathrm{~d}, J=8.46 \mathrm{~Hz})$ \\
\hline 5 & Lactate & $1.33(\mathrm{~d}, J=8.40 \mathrm{~Hz}), 4.11(\mathrm{q}, J=8.32 \mathrm{~Hz})$ \\
\hline 6 & Alanine & $1.48(\mathrm{~d}, J=8.57 \mathrm{~Hz}), 3.77(\mathrm{~m})$ \\
\hline 7 & Lysine & $1.72(\mathrm{~m}), 1.90(\mathrm{~m})$ \\
\hline 8 & $\mathrm{~N}$-acetyl glycoprotein & $2.04(s)$ \\
\hline 9 & Glutamate & $2.08(\mathrm{~m}), 2.35(\mathrm{~m}), 3.75(\mathrm{~m})$ \\
\hline 10 & $\beta$-hydroxybutyric & $2.34(\mathrm{dd})$ \\
\hline 11 & Pyroracemic & $2.37(\mathrm{~s})$ \\
\hline 12 & Glutamine & $2.45(\mathrm{~m})$ \\
\hline 13 & Citrate & $2.53(\mathrm{~d}, J=18.19 \mathrm{~Hz}), 2.65(\mathrm{~d}, J=18.19 \mathrm{~Hz})$ \\
\hline 14 & Creatine & $3.02(s)$ \\
\hline 15 & Creatinine & 3.04 (s), 3.91 (s) \\
\hline 16 & Choline & $3.21(\mathrm{~s}), 3.51(\mathrm{t}, \mathrm{J}=2.20 \mathrm{~Hz})$ \\
\hline 17 & Trimethylamine oxide & $3.26(\mathrm{~s})$ \\
\hline 18 & Glycine & $3.56(s)$ \\
\hline 19 & Threonine & $3.60(\mathrm{~d}, J=4.8 \mathrm{~Hz}), 4.24(\mathrm{~m})$ \\
\hline 20 & Aspartic & $3.90(\mathrm{dd})$ \\
\hline 21 & $\beta$-glucose & $4.65(\mathrm{~d}, J=7.80 \mathrm{~Hz})$ \\
\hline 22 & $\alpha$-glucose & $5.24(\mathrm{~d}, J=4.20 \mathrm{~Hz})$ \\
\hline
\end{tabular}

to HCs (17). Similarly, the OPLS-DA scores plot between the group of bipolar depression at baseline and the group of bipolar depression after 8 weeks medication was established (Figure 3C), which showed a clear separation between the two groups and were verified to be valid by the permutation testing (Figure 3D). The score plot of OPLS-DA model showed no significant difference between healthy controls and bipolar depression after 8 weeks medication, with some overlap. This indicates that the metabolite of BD after 8 weeks medication differs little from that of healthy controls (Figure 3E). The corresponding S-plot (Figure 3F) indicated that the lactate, choline, $\alpha$-glucose, $\beta$ glucose, $\mathrm{N}$-acetyl glycoprotein and trimethylamine oxide had the tendency to return to the normal levels after 8 weeks medication (VIP $>1$ and $p<0.05$ ), and especially the Lactate, choline and $\alpha$-glucose could be basically restored to normal levels. The changes of these 7 metabolites between the three groups were clearly shown in Figure 4. These results mean that effective medication had significant effects on plasma metabolites of bipolar depression.

\section{Pathways Analysis of Metabolites Related to Medication}

In order to further explore the mechanism of the efficacy of medication for bipolar depression, the metabolic pathways involved in these different metabolites related to the efficacy of medication were analyzed. The main metabolic pathways involved were: (1) pyruvate metabolism, (2) glycolysis or gluconeogenesis, (3) glycerophospholipid metabolism, (4) starch and sucrose metabolism, (5) pentose phosphate pathway, (6) propionate metabolism, (7) galactose metabolism, (8) glycine, 

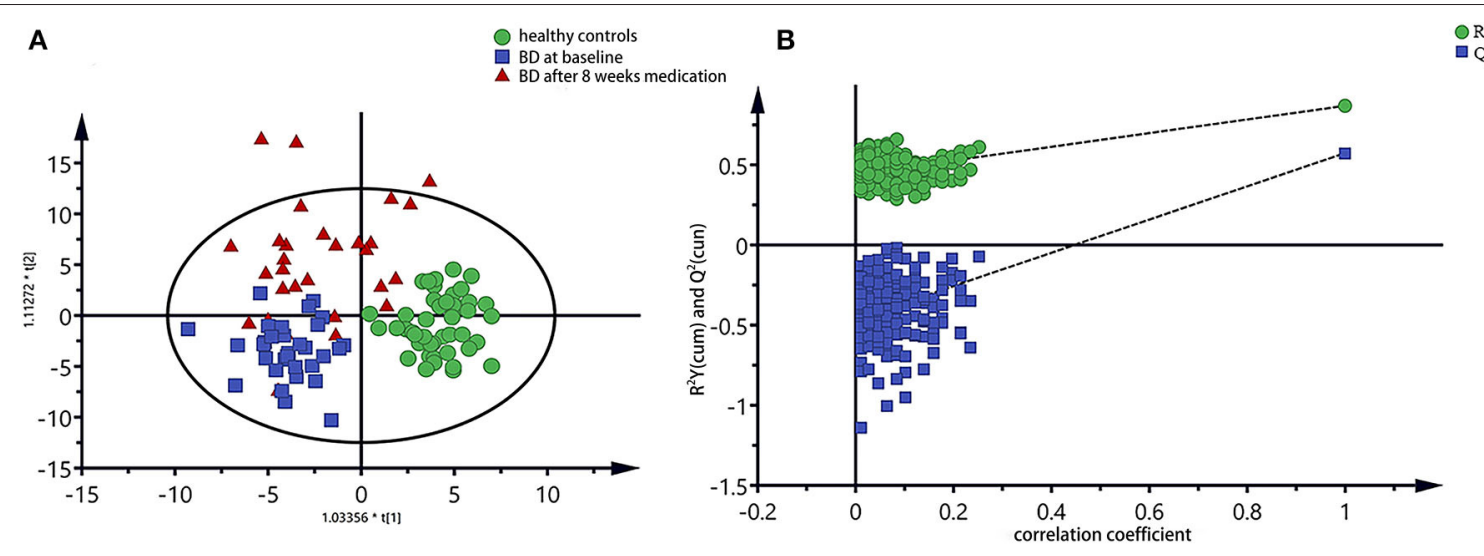

FIGURE 2 | PLS-DA model of all three groups of plasma spectra, (A) PLS-DA score plots of ${ }^{1} \mathrm{H}$ NMR spectra, in which each point represented a plasma metabolome of one subject, and the distance between data points reflected the scale of their metabolic differences; (B) 200-iteration permutation test map of the PLS-DA model.

serine and threonine metabolism, and (9) amino sugar and nucleotide sugar metabolism (Figure 5).

\section{DISCUSSION}

This study is a continuation of our group's research on blood diagnostic biomarkers for BD. We excluded patients who did not belong to bipolar II type and those who were not willing to participate in the follow-up. A total of 31 unmedicated patients in the acute stage of bipolar depression were recruited for 8 weeks of medication and follow-up. Encouragingly, the results of differential metabolites associated with bipolar depression were consistent with the previous results, which to some extent verified the reliability of our previous study results. As for the detection technique of metabolites profiles, we still chose a relatively mature ${ }^{1} \mathrm{H}$-NMR metabolomics technique. This single technique was able to detect a limited number of metabolites, and we tried to look for differential metabolites related to diagnosis and efficacy of medication of bipolar depression in these limited metabolites range.

The efficacy of medication was assessed according to the reduction rate of HAMD. In the absence of objective biological indicators for the assessment of mental illness, the HAMD scale, characterized by its relative objectivity and convenience, is the main clinical method for the assessment of depression severity and efficacy of medication. In this study, HAMD scores were used to help determine the acute phase and the effectiveness of medication in patients with bipolar depression. And the metabolite profiles were compared between the acute and significant improvement stages of bipolar depression based on HAMD score to look for changes of blood metabolites regardless of specific drug regimen.

The results of this study were that patients with bipolar depression showed relatively consistent changes in blood metabolites after 8 weeks medication. Therefore, we conclude that the intrinsic biological changes in significant improvement stage of bipolar depression are likely to be consistent despite the different medication regimens. Previous studies have reported that both lithium and valproate can reduce inositol levels in BD patients for therapeutic effects (22). These metabolites that change with significant improvement were related to energy metabolism (lactic acid, $\alpha$-glucose, $\beta$-glucose), choline, amino acid metabolism (glycine, $\mathrm{N}$-acetyl glycoprotein), and intestinal microbial metabolism (trimethylamine oxide), the levels of which had the tendency to return to the normal after 8 weeks medication. Only Glycine was reduced in both the acute and significant improvement stages of $\mathrm{BD}$, suggesting that glycine may be a characteristic marker of bipolar depression and does not change with the improvement of bipolar depression. These altered metabolites involved multiple metabolic pathways, and pyruvate metabolism was more closely related to therapeutic effect evaluation of $\mathrm{BD}$ (pathway impact $>0.1$, $p<0.05)$.

Energy metabolism is one of the most important metabolic ways in the body. In this study, we observed significant decreases in $\alpha$-glucose and $\beta$-glucose levels and significant increases in lactic acid levels in patients with bipolar depression at baseline. After 8 weeks medication, the levels of $\alpha$-glucose and lactic acid were basically normal, and the level of $\beta$-glucose also has the tendency to return to normal. Glucose is the main source of energy in the body, and its aerobic oxidation is the main way of energy supply. In the absence of oxygen supply, sugar goes through anaerobic oxidation to produce lactic acid, leading to a decline in energy supply (23). Therefore, the results of this study suggested that the anaerobic oxidation pathway was enhanced and the aerobic oxidation pathway was inhibited in patients with bipolar depression. This energy metabolism disorder in bipolar depression may explain the symptoms of fatigue, poor energy and slow thinking. Drug therapy can regulate energy metabolism and restore it to normal. Energy homeostasis imbalances of BD have been previously reported $(24,25)$.

Choline is an important substance in the body, which is the main synthetic raw material of glycerol phospholipids and can also be used in the synthesis of acetylcholine, which is the important neurotransmitter (26). In this study, patients with 


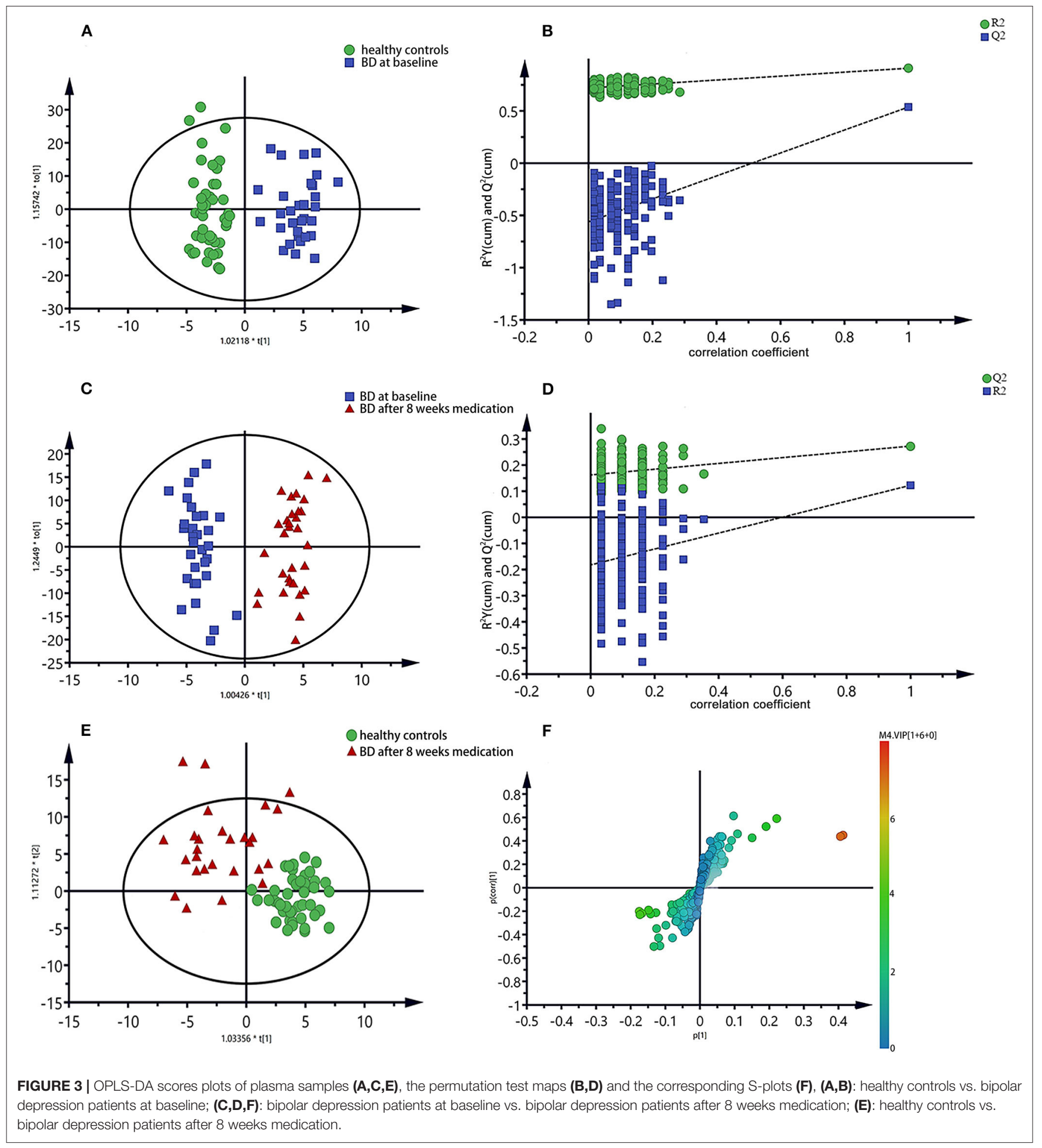

bipolar depression had reduced choline levels and returned to normal after 8 weeks of medication. Reduced choline levels in patients with bipolar depression can lead to reduced production of glycerophospholipids and acetylcholine, both of which are important functional substances. Effective drug therapy could improve biological function by increasing blood choline levels. Previous studies have found abnormal levels of choline in BD (27). Therefore, serum choline level is expected to be a biomarker for the diagnosis and the evaluation of drug treatment effectiveness of bipolar depression. 

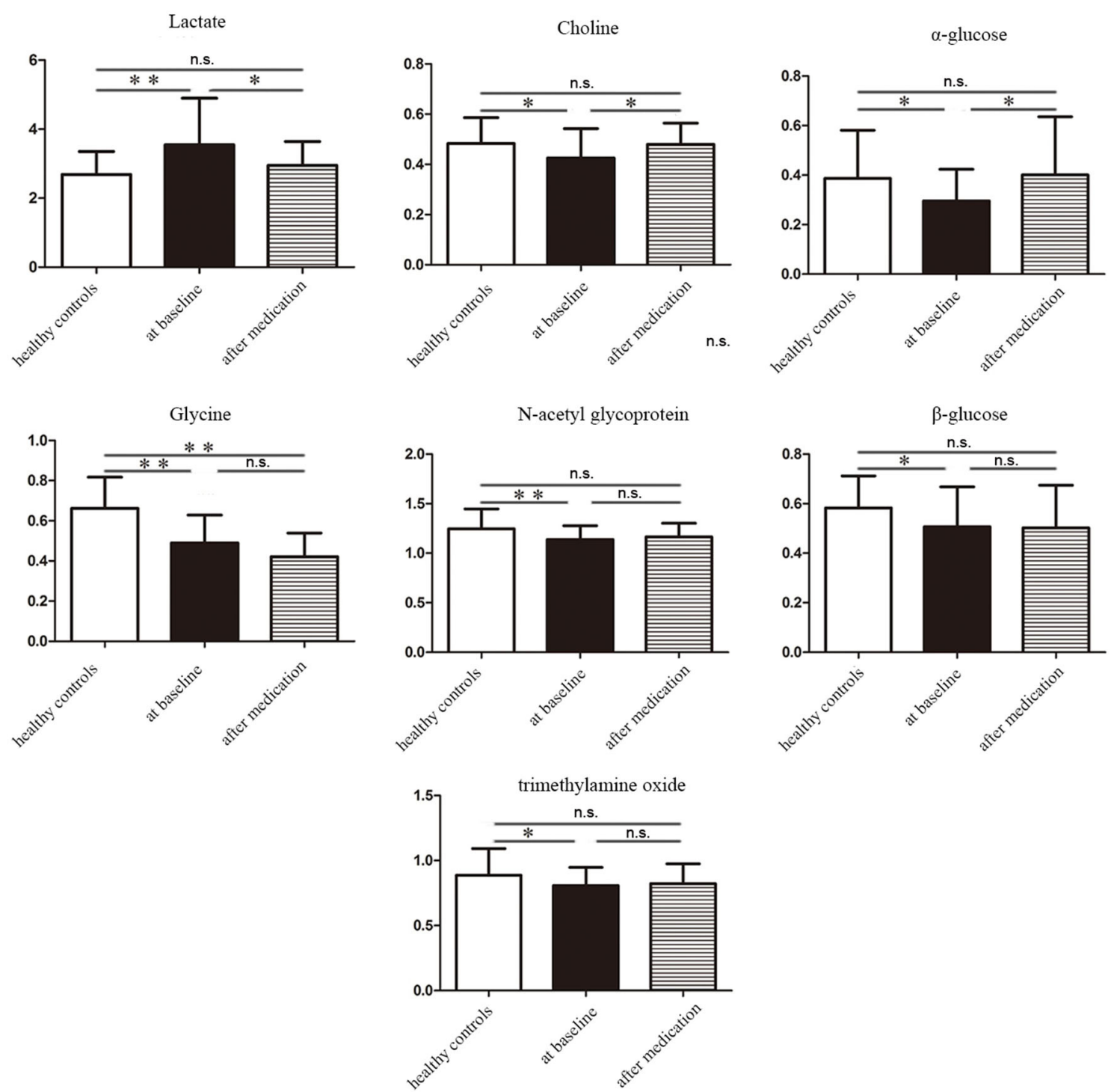

FIGURE 4 | The concentration variation diagram of the 7 different metabolites among healthy controls, bipolar depression patients at baseline and bipolar depression patients after 8 weeks medication. n.s., non-significant ${ }^{\star} P<0.05$, ${ }^{\star \star} P<0.01$.

This study found that patients with bipolar depression had lower glycine levels than HCs, and glycine levels continued to decrease after effective medication. The results indicated that glycine may be a characteristic marker of bipolar depression and does not change with the improvement of bipolar depression. Glycine can affect the excitability of the nervous system (28, 29). It has been reported that glycine, serine and threonine have obvious changes in serum of patients with refractory depression (30). Changes in glycine levels were also found in the cerebrospinal fluid of patients with BD (31). Therefore, it can be speculated that glycine is abnormal in some mental disorders, not just bipolar disorder and may be involved in the pathological mechanism of psychiatric diseases. The study also found that baseline levels of $\mathrm{N}$-acetylamino glycoprotein were lower in patients with bipolar depression than in HCs, and returned to normal after medication. Due to the single technology in this study, n-acetylamino glycoprotein classification could not be carried out, so future research could improve on this.

We also found elevated trimethylamine oxide levels in patients with bipolar depression, which returned to normal after 8 weeks of medication. Trimethylamine oxide is a metabolite related to intestinal flora, and its production cannot be separated from the involvement of intestinal flora. Intestinal flora is closely related to human health (32). Researchers have found that gut flora can affect human brain function through the microbiota-gut-brain axis (33). Several studies in recent years have reported abnormal intestinal flora in BD (34-36). Combined with these findings, 


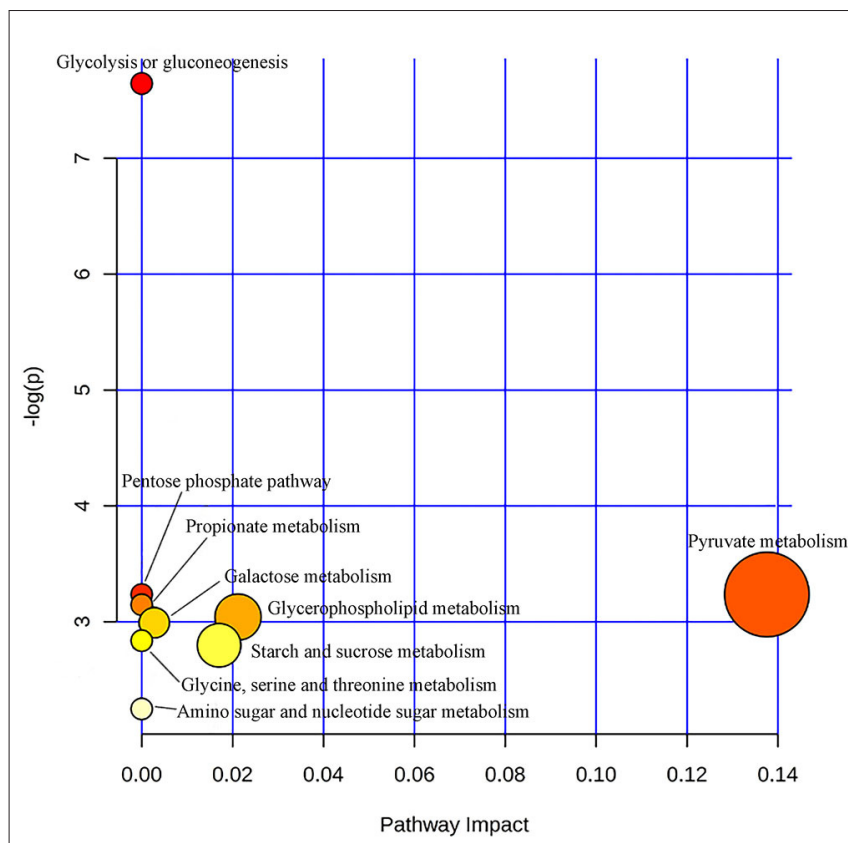

FIGURE 5 | Metabolic pathways analysis map of the metabolites related to the efficacy of medication for bipolar depression. Color intensity (white to red) reflects increasing statistical significance, while circle diameter covaries with pathway impact. of drug treatment effectiveness of bipolar depression. Lactate was elevated during the acute phase of bipolar depression and basically returned to normal levels after 8 weeks of effective medication. Levels of Choline, $\alpha$-glucose, $\beta$-glucose, $\mathrm{N}$-acetyl glycoprotein and trimethylamine oxide all decreased in the acute phase of bipolar depression and returned to normal levels to varying degrees in significant improvement stage. Glycine continued to decrease at baseline and after 8 weeks medication of bipolar depression. Therefore, it can be speculated that glycine may be a characteristic marker of bipolar depression and does not change with the improvement of bipolar depression, while lactate, choline, $\alpha$-glucose, $\beta$-glucose, $\mathrm{N}$-acetyl glycoprotein and trimethylamine oxide may be biomarkers associated with the pathological development of bipolar depression and can reflect the severity of the illness. These identified metabolites were associated with multiple metabolic pathways, especially pyruvate metabolism. Moreover, these metabolites are involved in energy metabolism, amino acid metabolism and intestinal microbial metabolism, which were consistent with some previously reported studies. Metabolomics has a great development prospect in the study of biological indicators related to the efficacy of BD. It is hoped that more studies in this field with a larger sample size will be carried out in the future, so as to further explore the internal mechanism of the course evolution of BD.

\section{DATA AVAILABILITY STATEMENT}

The original contributions presented in the study are included in the article/supplementary material, further inquiries can be directed to the corresponding author/s.

\section{ETHICS STATEMENT}

The studies involving human participants were reviewed and approved by the Medical Ethics Committee of Shanxi Bethune Hospital. The patients/participants provided their written informed consent to participate in this study.

\section{AUTHOR CONTRIBUTIONS}

YR conceived and designed the experiments. JJ, X-HC, SB, FY, L-NM, and X-XC collected subjects. PW performed the experiments and analyzed the data. X-JG, PW, and YR wrote and revised the manuscript. All authors contributed to the article and approved the submitted version.

\section{FUNDING}

This work was supported by the National Natural Science Foundation of China (8210053813), Applied Basic Research Projects of Shanxi Province, China (201901D111418), Research Project Supported by Shanxi Scholarship Council of China (2021167). 


\section{REFERENCES}

1. Post RM, Altshuler LL, Kupka R, McElroy SL, Frye MA, Rowe M, et al. Age of onset of bipolar disorder: combined effect of childhood adversity and familial loading of psychiatric disorders. J Psychiatr Res. (2016) 81:6370. doi: 10.1016/j.jpsychires.2016.06.008

2. Rowland TA, Marwaha S. Epidemiology and risk factors for bipolar disorder. Therap Adv Psychopharmacol. (2018) 8:25169. doi: $10.1177 / 2045125318769235$

3. Vázquez GH, Holtzman JN, Lolich $M$, Ketter TA, Baldessarini RJ. Recurrence rates in bipolar disorder: systematic comparison of long-term prospective, naturalistic studies versus randomized controlled trials. Eur Neuropsychopharmacol. (2015) 25:150112. doi: 10.1016/j.euroneuro.2015.07.013

4. Moradi M, Saidijam M, Yadegarazari R, Jahangard L, Seifi M, Matinnia N, et al. Genes encoding GABA- $\beta$ and HT1D receptors in bipolar I (manic phase) patients. Basic Clin Neurosci. (2018) 9:129-34. doi: 10.29252/nirp.bcn.9. 2.129

5. Ascoli BM, Géa LP, Colombo R, Barbé-Tuana FM, Kapczinski F, Rosa AR. The role of macrophage polarization on bipolar disorder: identifying new therapeutic targets. Aust N Z J Psychiatry. (2016) 50:618-30. doi: $10.1177 / 0004867416642846$

6. Frangou S. Neuroimaging markers of risk, disease expression, and resilience to bipolar disorder. Curr Psychiatry Rep. (2019) 21:52. doi: 10.1007/s11920-019-1039-7

7. Fries GR, Vasconcelos-Moreno MP, Gubert C, dos Santos BT, Sartori J, Eisele B, et al. Hypothalamic-pituitary-adrenal axis dysfunction and illness progression in bipolar disorder. Int J Neuropsychopharmacol. (2014) 18:43. doi: 10.1093/ijnp/pyu043

8. Jaworska-Andryszewska P, Rybakowski J. Negative experiences in childhood and the development and course of bipolar disorder. Psychiatr Pol. (2016) 50:989-1000. doi: 10.12740/PP/61159

9. Harwood AJ. Lithium and bipolar mood disorder: the inositoldepletion hypothesis revisited. Mol Psychiatry. (2005) 10:11726. doi: $10.1038 /$ sj.mp. 4001618

10. O’Donnell T, Rotzinger S, Nakashima TT, Hanstock CC, Ulrich M, Silverstone PH. Chronic lithium and sodium valproate both decrease the concentration of myoinositol and increase the concentration of inositol monophosphates in rat brain. Eur Neuropsychopharmacol. (2003) 13:199207. doi: 10.1016/S0924-977X(03)00011-7

11. Rattray NJW, Deziel NC, Wallach JD, Khan SA, Vasiliou V, Ioannidis JPA, et al. Beyond genomics: understanding exposotypes through metabolomics. Hum Genomics. (2018) 12:4. doi: 10.1186/s40246-0180134-x

12. Ribeiro HC, Klassen A, Pedrini M, Carvalho MS, Rizzo LB, Noto $\mathrm{MN}$, et al. A preliminary study of bipolar disorder type I by mass spectrometry-based serum lipidomics. Psychiatry Res. (2017) 258:26873. doi: 10.1016/j.psychres.2017.08.039

13. Chen JJ, Liu Z, Fan SH, Yang DY, Zheng P, Shao WH, et al. Combined application of NMR- and GC-MS-based metabonomics yields a superior urinary biomarker panel for bipolar disorder. Sci Rep. (2014) 4:5855. doi: 10.1038/srep05855

14. Yoshimi N, Futamura T, Bergen SE, Iwayama Y, Ishima T, Sellgren $\mathrm{C}$, et al. Cerebrospinal fluid metabolomics identifies a key role of isocitrate dehydrogenase in bipolar disorder: evidence in support of mitochondrial dysfunction hypothesis. Mol Psychiatry. (2016) 21:150410. doi: $10.1038 / \mathrm{mp} .2015 .217$

15. Lan MJ, McLoughlin GA, Griffin JL, Tsang TM, Huang JT, Yuan P, et al. Metabonomic analysis identifies molecular changes associated with the pathophysiology and drug treatment of bipolar disorder. Mol Psychiatry. (2009) 14:269-79. doi: 10.1038/sj.mp.4002130

16. Tondo L, Vázquez GH, Baldessarini RJ. Depression and mania in bipolar disorder. Curr Neuropharmacol. (2017) 15:353-8. doi: 10.2174/1570159X146661606062 10811

17. Ren Y, Bao S, Jia Y, Sun XL, Cao XX, Bai XY, et al. Metabolic profiling in bipolar disorder patients during depressive episodes. Front Psychiatry. (2020) 11:569612. doi: $10.3389 /$ fpsyt.2020.569612
18. Yatham LN, Kennedy SH, Parikh SV, Schaffer A, Bond DJ, Frey BN, et al. Canadian Network for Mood and Anxiety Treatments (CANMAT) and International Society for Bipolar Disorders (ISBD) 2018 guidelines for the management of patients with bipolar disorder. Bipolar Disord. (2018) 20. doi: 10.1111/bdi.12609

19. Liu CC, Wu YF, Feng GM, Gao XX, Zhou YZ, Hou WJ, et al. Plasma-metabolite-biomarkers for the therapeutic response in depressed patients by the traditional Chinese medicine formula Xiaoyaosan: a (1)H NMR-based metabolomics approach. J Affect Disord. (2015) 185:15663. doi: 10.1016/j.jad.2015.05.005

20. Kurita M, Nishino S, Numata Y, Okubo Y, Sato T. The noradrenaline metabolite MHPG is a candidate biomarker between the depressive, remission, and manic states in bipolar disorder I: two longterm naturalistic case reports. Neuropsychiatr Dis Treat. (2015) 11:353-8. doi: 10.2147/NDT.S74550

21. Bujak R, Struck-Lewicka W, Markuszewski MJ, Kaliszan R. Metabolomics for laboratory diagnostics. J Pharm Biomed Anal. (2015) 113:10820. doi: 10.1016/j.jpba.2014.12.017

22. Yu W, Greenberg ML. Inositol depletion, GSK3 inhibition and bipolar disorder. Future Neurol. (2016) 11:135-48. doi: 10.2217/fnl-2016-0003

23. Valenza F, Aletti G, Fossali T, Chevallard G, Sacconi F, Irace M, et al. Lactate as a marker of energy failure in critically ill patients: hypothesis. Critical Care (London, England). (2005) 9:588-93. doi: 10.1186/cc3818

24. Yoshimi N, Futamura T, Kakumoto K, Salehi AM, Sellgren CM, HolménLarsson J, et al. Blood metabolomics analysis identifies abnormalities in the citric acid cycle, urea cycle, and amino acid metabolism in bipolar disorder. BBA Clin. (2016) 5:151-8. doi: 10.1016/j.bbacli.2016.03.008

25. Mansur RB, Lee Y, McIntyre RS, Brietzke E. What is bipolar disorder? A disease model of dysregulated energy expenditure. Neurosci Biobehav Rev. (2020) 113:529-45. doi: 10.1016/j.neubiorev.2020.04.006

26. Sussulini A, Prando A, Maretto DA, Poppi RJ, Tasic L, Banzato CE, et al. Metabolic profiling of human blood serum from treated patients with bipolar disorder employing 1H NMR spectroscopy and chemometrics. Anal Chem. (2009) 81:9755-63. doi: 10.1021/ac901502j

27. Galińska-Skok B, Małus A, Konarzewska B, Rogowska-Zach A, Milewski R, Tarasów E, et al. Choline compounds of the frontal lobe and temporal glutamatergic system in bipolar and schizophrenia proton magnetic resonance spectroscopy study. Dis Markers. (2018) 2018:3654894. doi: 10.1155/2018/3654894

28. Hernandes MS, Troncone LR. Glycine as a neurotransmitter in the forebrain: a short review. J Neural Trans (Vienna, Austria: 1996). (2009) 116:155160. doi: 10.1007/s00702-009-0326-6

29. Dohi T, Morita K, Kitayama T, Motoyama N, Morioka N. Glycine transporter inhibitors as a novel drug discovery strategy for neuropathic pain. Pharmacol Ther. (2009) 123:54-79. doi: 10.1016/j.pharmthera.2009.03.018

30. Maes M, Verkerk R, Vandoolaeghe E, Lin A, Scharpé S. Serum levels of excitatory amino acids, serine, glycine, histidine, threonine, taurine, alanine and arginine in treatment-resistant depression: modulation by treatment with antidepressants and prediction of clinical responsivity. Acta Psychiatr Scand. (1998) 97:302-8. doi: 10.1111/j.1600-0447.1998.tb10004.x

31. Pålsson E, Jakobsson J, Södersten K, Fujita Y, Sellgren C, Ekman CJ, et al. Markers of glutamate signaling in cerebrospinal fluid and serum from patients with bipolar disorder and healthy controls. Eur Neuropsychopharmacol. (2015) 25:133-40. doi: 10.1016/j.euroneuro.2014.11.001

32. Zhou B, Yuan Y, Zhang S, Guo C, Li X, Li G, et al. Intestinal flora and disease mutually shape the regional immune system in the intestinal tract. Front Immunol. (2020) 11:575. doi: 10.3389/fimmu.2020.00575

33. Clarke G, Grenham S, Scully P, Fitzgerald P, Moloney RD, Shanahan F, et al. The microbiome-gut-brain axis during early life regulates the hippocampal serotonergic system in a sex-dependent manner. Mol Psychiatry. (2013) 18:666-73. doi: $10.1038 / \mathrm{mp} .2012 .77$

34. Evans SJ, Bassis CM, Hein R, Assari S, Flowers SA, Kelly MB, et al. The gut microbiome composition associates with bipolar disorder and illness severity. J Psychiatr Res. (2017) 87:23-9. doi: 10.1016/j.jpsychires.2016.12.007

35. Painold A, Mörkl S, Kashofer K, Halwachs B, Dalkner N, Bengesser S, et al. A step ahead: exploring the gut microbiota in inpatients with bipolar disorder during a depressive episode. Bipolar Disord. (2019) 21:409. doi: $10.1111 /$ bdi. 12682 
36. Guo L, Ji C, Ma Q, Fan Y, Feng J, Chen C, et al., editors. The diversity and the abundance of gut microbiome in patients with bipolar disorder. Chin J Psychiatry. (2018) 51:98-104. doi: 10.3760/cma.j.issn.1006-7884.2018. 02.005

Conflict of Interest: The authors declare that the research was conducted in the absence of any commercial or financial relationships that could be construed as a potential conflict of interest.

Publisher's Note: All claims expressed in this article are solely those of the authors and do not necessarily represent those of their affiliated organizations, or those of the publisher, the editors and the reviewers. Any product that may be evaluated in this article, or claim that may be made by its manufacturer, is not guaranteed or endorsed by the publisher.

Copyright (C) $2021 \mathrm{Guo}, \mathrm{Wu}, \mathrm{Cui}$, Jia, Bao, Yu, Ma, Cao and Ren. This is an openaccess article distributed under the terms of the Creative Commons Attribution License (CC BY). The use, distribution or reproduction in other forums is permitted, provided the original author(s) and the copyright owner(s) are credited and that the original publication in this journal is cited, in accordance with accepted academic practice. No use, distribution or reproduction is permitted which does not comply with these terms. 